\title{
OBSERVATION OF GAP ACCEPTANCE DURING INTERSECTION APPROACH
}

\author{
Delphine Cody, Christopher Nowakowski, Bénédicte Bougler \\ California PATH program - Institute of Transportation Studies \\ University of California, Berkeley \\ Richmond, California, U.S.A. \\ E-mail: cody@path.berkeley.edu
}

\begin{abstract}
Summary: This paper presents the results of a field test evaluating drivers' decision making and gap acceptance for a left turn maneuver with opposite traffic; with a focus on the scenario where the turning vehicle is approaching the intersection. Twenty-three participants drove an instrumented vehicle ten times around an extended block turning left at four specific intersections. DGPS and radar data were combined to represent the trajectories of the turning vehicle and opposite traffic and to compute the gap between the turning vehicle and the lead vehicle of the on-coming traffic. The results cover the categorization of driver behavior based on the velocity profile while crossing the intersection. The velocity profile shows whether a driver stopped during the maneuver and the categories are defined based on where the driver stopped. The trajectories were then further sorted to account for the difference in speed profile or stopping location within one category. The characteristics of the accepted and rejected lags are presented and discussed, with an emphasis on turning drivers' speed adaptation to the presence of an on-coming vehicle, and the presence of following traffic on a decision to stop. The conclusion of this study is that although valuable information was gathered during this field test, the data collection setup did not capture sufficiently the characteristic of the opposite traffic for a quantitative description of the effect of subsequent gaps on drivers' decision to stop. Therefore, further data collection will be conducted on an instrumented intersection on a closed track.
\end{abstract}

\section{INTRODUCTION AND OBJECTIVES}

Left Turn Across Path/Opposite Direction (LTAP/OD) crashes are the most prevalent conflicts at signalized intersections, resulting in nearly 18 percent of crashes (Ragland and Zabyshny, 2003). This scenario most commonly occurs at signalized intersections with a permissive green for left turn. The Infrastructure Consortium, consisting of the States of California, Minnesota and Virginia, in partnership with the US DOT's Federal Highway Administration, sponsored the Intersection Decision Support (IDS) project in order to seek an Intelligent Transportation solution for this type of crash.

Previous work on gap acceptance for left turn involved the identification of parameter(s) used by drivers to accept or reject a gap (Hancock et al., 1991) with a focus on scenarios involving a stopped SV (Alexander et al., 2002; Staplin, 1995). Chovan et al. (1994) stressed the need to address the scenario of a moving Subject Vehicle (SV) in their efforts to model gap acceptance. These authors propose distinguishing between two turning behaviors: (i) the driver turns without 
stopping and (ii) the driver stops before to turn. Gap acceptance for drivers stopped prior to turn has been heavily investigated. However, less data are available for the situation of drivers turning without stopping. Chovan et al. (1994) referenced the results from Ueno and Ochiai (1993), where the stop/no stop decision relies on the time gap between the Principal Other Vehicle (POV) and the SV turning path when the SV is at the start of the turn. The threshold for stopping is defined as a time gap less than 3 seconds, with no drivers stopping if the time gap exceeds 8 seconds. Between these two boundaries, the acceptance is a positive function of the gap, i.e., the longer the gap the higher the acceptance rate.

For the purpose of designing a countermeasure, more information, and especially quantitative data, was necessary to obtain in order to: (i) specify a decision zone, i.e., the range of time during which to provide a message to the driver, the earlier being near the decision point and the latest depending on drivers' possibility of stopping prior to entering the POV path, which also supports the design of the message provided (Cody, 2005; Nowakowski, 2006) and (ii) quantify the gaps corresponding to the decisions.

In terms of gap, Brilon (1999) distinguished gap, the time interval between the arrival of two successive vehicles; from lag, the period of time between the SV and POV arrival at the intersection. Moreover, the relevant parameter to measure is the "projected lag," corresponding to the scene when the driver is approaching the intersection and making the decision to turn or not in front of an on-coming POV, rather than the actual lag, which results from the driver's decision.

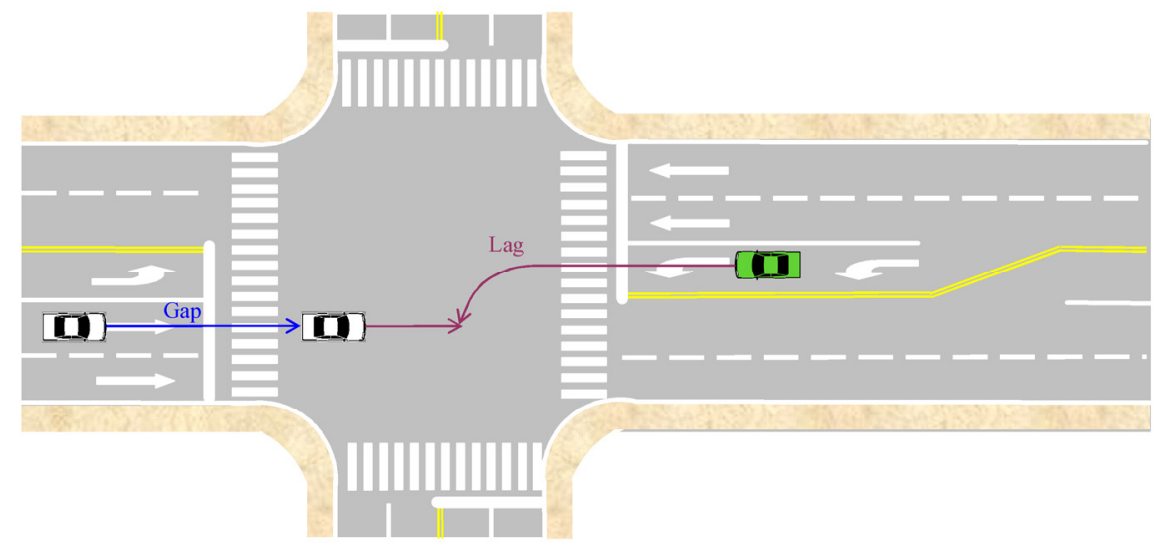

Figure 1. Difference between lag and gap

Figure 1 above illustrates the difference between a gap, measured as the time or distance between two vehicles traveling in the same direction, and the lag, which represents the intersection of two vehicles trajectories in time or distance.

This paper is organized as follows. The first section presents the methods used to conduct the study. The second section describes gap acceptance as measured in a field test and a discussion of the method used for measuring gaps between a moving SV and POV. We conclude with the insights this data brings to the development of an Intersection Decision Support system for LTAP/OD crashes and outline future research plans. 


\section{METHODS}

A field study was conducted in an urban environment. Twenty-three drivers, ages 25 to 75 , drove an instrumented vehicle ten times around a route including four intersections, turning left at each intersection. The instrumentation recorded drivers' manipulation of vehicle controls (throttle, brake, and turn signal), vehicle motion (speed, acceleration, and position using DGPS), distance and relative velocity with front and on-coming traffic (radars), and video with a 5-channel recorder (driver's face, 3 front scenes, and rear scene). The DGPS and radar data were combined in order to render the SV and POV trajectories relative to the zone of conflict (ZOC), where the trajectories would collide. This zone corresponds to a square delimited by the solid lines on the road where the SV and POV are approaching and the solid line from the SV's destination lane.

A post-hoc calculation was used to estimate the lag. Figure 2 below illustrates the case of an accepted lag (graph on the left) and a rejected lag (right figure). The $\mathrm{x}$ axis is the time at which the data was captured; the y axis represents the distance to the zone of conflict. As the SV and POV are traveling in opposite directions, the SV distance to the zone is negative while the POV distance is positive. The radar data gathered from the SV is incomplete, either because an oncoming vehicle fell off the radar's field of view or because other vehicles occluded the field of view.
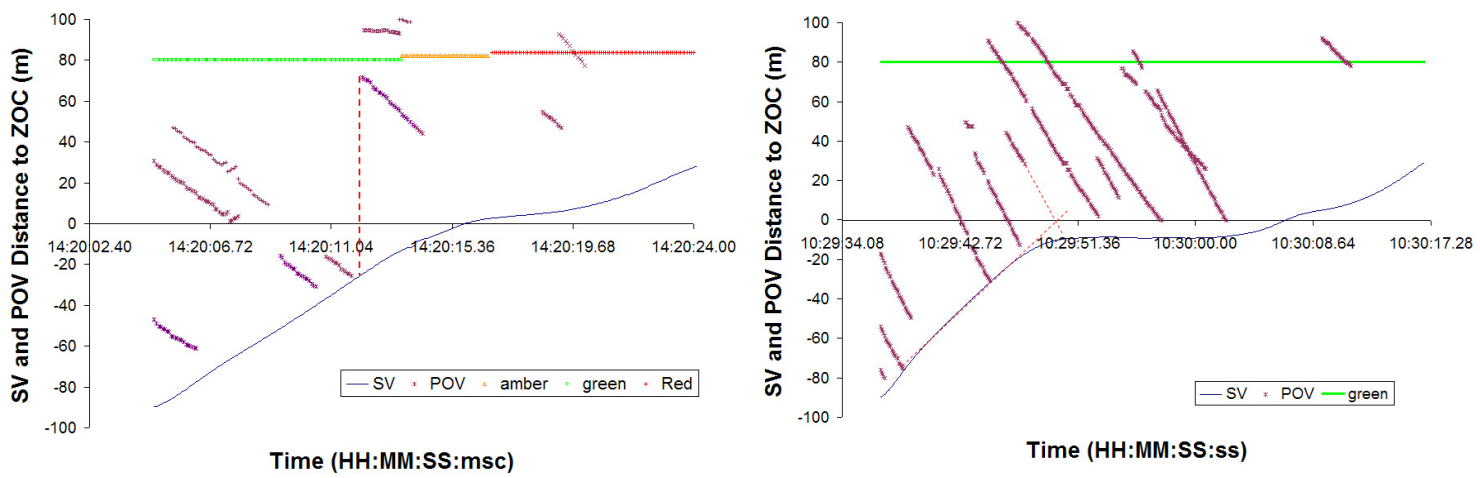

Figure 2. Example of accepted and rejected lag

On the graph on the left, the dashed vertical line represents the time at which the lag is determined. In this case, the SV was $-2.8 \mathrm{sec}$ before the Zone of conflict, while the POV was at $5.8 \mathrm{sec}$ from the same zone. On the figure on the right, the dashed lines illustrate how in some situations there is a need to extrapolate a POV trajectory in order to determine with which vehicle there is a conflict.

The prime independent measure used for this study was the driver response to a lag, i.e., whether or not the driver chooses to cross in front of POV. The prime dependant measure was the lag, measured in time or distance. 


\section{RESULTS}

Data was collected for 920 intersection crossings, and 913 were included in the analysis, the DGPS data quality was too poor to use for seven cases. The results first introduce the intersection crossings categories and then the characteristics of accepted and rejected lags.

\section{Driver behavior categorization}

The intersection crossings were categorized into four groups based on the SV driver's trajectory and stopping position relative to the intersection. For trajectory 1, the SV driver turns without stopping prior or in the intersection; for trajectory 2, the SV driver stops prior to the stop line/crosswalk boundary, and crosses the intersection. For trajectory 3, the SV driver stops prior to the stop line and again in the intersection, and for trajectory 4, the SV driver stops once in the intersection.

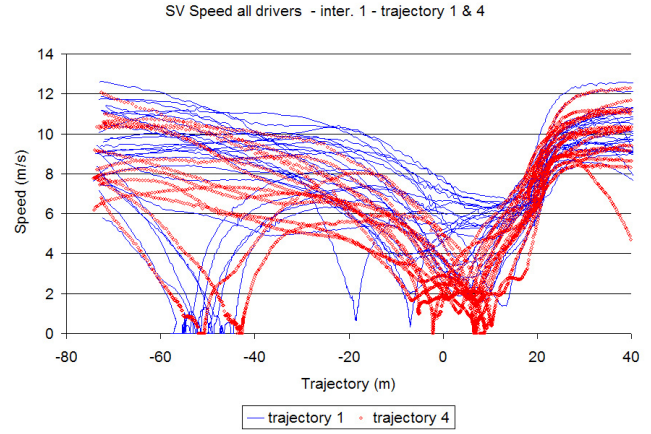

Figure 3. Trajectories 1 and 4 for one intersection based on speed profile

Figure 3 illustrate the difference in speed profile between trajectories 1 and 4 . Several stops occurred between 60 to 40 meters from the stop line. These cases resulted from the SV reaching the end of a platoon clearing the intersection when the light turns green.

\section{Gap Acceptance}

We gathered 21 cases of accepted lag with a distant POV and 39 rejected lags, i.e., the drivers did not turn in front of the POV, of which 17 led to a slow down and the SV turning after the POV without stopping and 22 led to a stop.
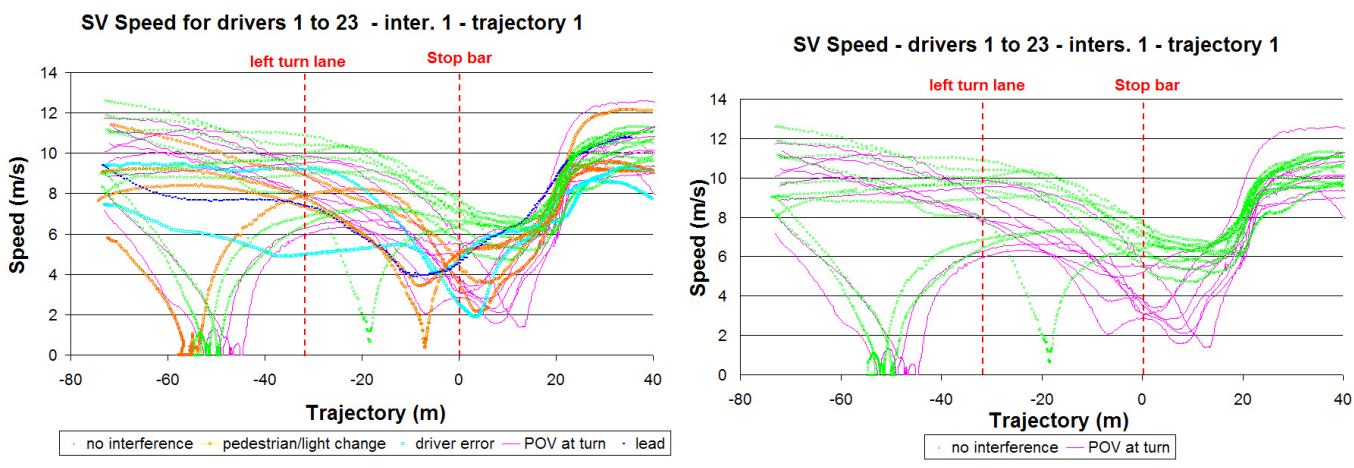

Figure 4. Refinement of trajectory 1 categorization 
Figure 4 illustrates the refinement of categorization of trajectory 1 for one of the observed intersections; the same sorting was operated for each intersection, but on different graphs as the $\mathrm{x}$ axis represents the trajectory along a specific intersection. The turning speed varies between 2 and $6 \mathrm{~m} / \mathrm{s}$ (graph on the left), the sorting of trajectory based on events, such as the presence of a lead, error committed by driver, presence of a POV. When looking only at cases where there was a POV or no interferences (graph on the right), it is possible to see the impact of the presence of a POV on the speed profile at the turning time.

Accepted lag. The table below presents the characteristics of the 21 cases of accepted lag. We provide the distance and time to ZOC for SV and POV when the lag was computed (i.e., when POV is sensed by the radar). The two last lines describe the lag in terms of time and distance. A negative difference means that the SV would clear the zone of conflict before the POV; a positive difference means that the POV would clear the zone of conflict before the SV.

Table 1. Characteristics of accepted lag not generating a slow down

\begin{tabular}{|l|c|c|c|c|}
\hline \multicolumn{1}{|c|}{ Parameter } & Minimum & Maximum & Mean & Std. Deviation \\
\hline POV Distance to ZOC (meters) & 49.00 & 113.00 & 85.61 & 18.09 \\
\hline POV time to ZOC (seconds) & 4.30 & 16.00 & 8.04 & 2.74 \\
\hline SV Distance to ZOC & 10.00 & 50.00 & 23.09 & 11.62 \\
\hline SV Time to ZOC & 2.10 & 6.30 & 3.76 & 1.08 \\
\hline Lag in time & -12.30 & 1.40 & -4.27 & 3.06 \\
\hline Lag in distance & -98.00 & -20.00 & -62.52 & 24.21 \\
\hline
\end{tabular}

Although all of the lags in terms of distance are negative, 2 lags in time are positive. This is due to cases where the POV was very close to the intersection and in the process of accelerating. These cases illustrate that the lag computation, for situations where the SV is moving toward the intersection, is a very dynamic process. If using the lag time as an indication of the spare time available to make the turn, then the mean could be used as an indicator

Slowing down to turn after POV. A total of 17 cases for which the SV driver slowed down more than required for the turn but did not stop have been identified. Out of these 17 cases, 15 could be processed. Their characteristics are presented below.

Table 2. Characteristics of lag generating a slow down but not a stop

\begin{tabular}{|l|c|c|c|c|}
\hline \multicolumn{1}{|c|}{ Parameter } & Minimum & Maximum & Mean & Std. Deviation \\
\hline POV Distance to ZOC (meters) & 18.00 & 82.00 & 54.93 & 20.83 \\
\hline POV time to ZOC (seconds) & 2.80 & 8.50 & 5.20 & 1.55 \\
\hline SV Distance to ZOC & 27.00 & 62.00 & 39.667 & 11.44 \\
\hline SV Time to ZOC & 3.20 & 6.70 & 4.93 & .96 \\
\hline Lag in time & -2.70 & 2.60 & -.27 & 1.33 \\
\hline Lag in distance & -42.00 & 19.00 & -15.26 & 18.75 \\
\hline
\end{tabular}

Table 2 is very similar to Table 1 . It is interesting to compare the mean time lag between this scenario and the preceding one. In this scenario, the mean lag time is very close to 0 and corresponds to situations where there was virtually no spare time. 
Stop for on-coming traffic. We identified 74 cases where the SV driver stopped within the intersection box: 32 are lags, i.e., the space is between the SV and POV, 25 were caused by platoons of waiting POV clearing the intersection, a situation that happens if the light turns green as the SV driver reaches the intersection, and the rest were marginal factors. Out of the 32 lags, 10 were considered special cases, for reasons such as light change or POV turning left when reaching the intersection, and not representing a threat anymore. In Table 3, the rejected mean lag is -.45 seconds (the SV would have cleared the ZOC a half second prior to the POV).

Table 3. Characteristics of rejected lags leading to a stop

\begin{tabular}{|l|c|c|c|c|c|}
\hline Parameter & $\mathrm{N}$ & Minimum & Maximum & Mean & Std. Deviation \\
\hline Lag $(\mathrm{m})$ & 32 & -70.00 & 56.00 & -24.81 & 23.65 \\
\hline Lag $(\mathrm{sec})$ & 32 & -7.90 & 7.00 & -.45 & 2.49 \\
\hline
\end{tabular}

The results indicated a significant difference between the lags that were accepted (mean -4.3 , std 2.2) and the lags that were rejected (mean -0.3, std 1.6). Half of the rejected lags led the SV driver to slow down and turn after the POV. For the other half, the driver eventually stopped in the middle of the intersection, because the gaps between the first POV and subsequent POVs were also rejected.

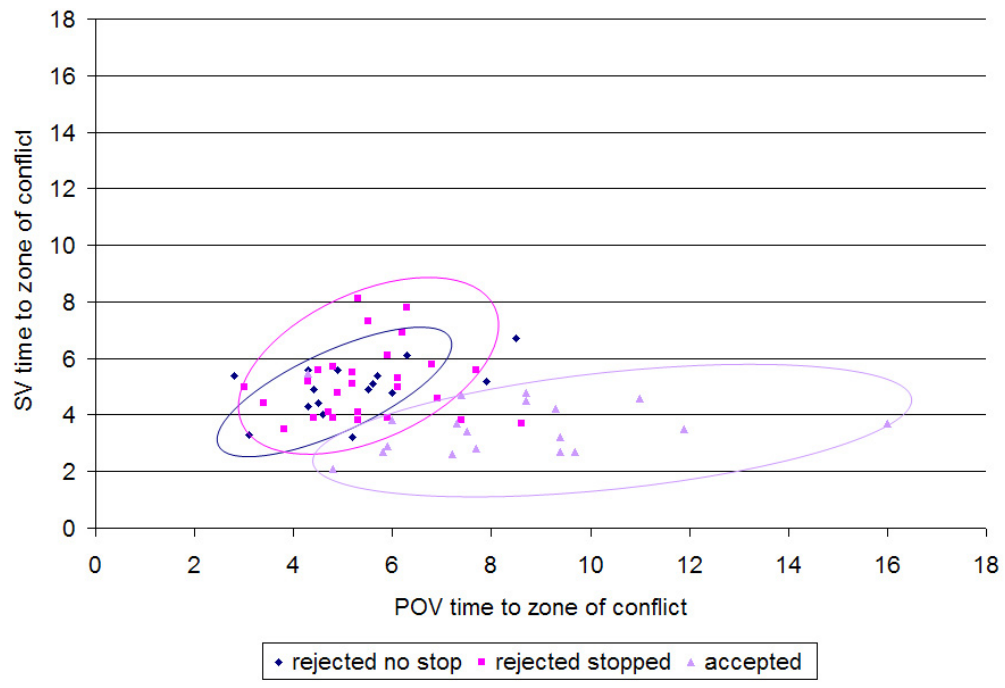

Figure 5. Clustering of accepted and rejected lags

Figure 5 above illustrates the clustering of lags, with the POV time to the zone of conflict on the $\mathrm{x}$ axis and the $\mathrm{SV}$ time to $\mathrm{ZOC}$ on the $\mathrm{y}$ axis. A similar pattern can be identified for the two categories of rejected lags, with time for SV and POV very close to each other, while the SV is closer to the ZOC than the POV for the accepted gap. This graph shows that the size of the lag is not the only predictor for determining whether a driver will stop or not, and that other variables are playing a role, such as the presence of a vehicle after the lead POV, and the gap between these two vehicles. 


\section{CONCLUSIONS}

The results analysis supported the proposed method for assessing lag acceptance for the scenario of the moving SV, although it also illustrated the shortcomings of vehicle-based data collection. Further studies will be conducted on an instrumented test track in order to improve the quality of the data and support the understanding of gap acceptance with a systematic testing. as well as the effect of the other factors influencing gap acceptance, such as the size and number of gaps following the lag, and its possible effect on more aggressive behavior.

Open questions that need to be discussed by the community are i) how to standardize the variable lag and gaps, so that data can be compared from different data collections, and ii) how to conduct field tests or naturalistic data collection at intersections where the measurements request a full view of the traffic situation that cannot be acquired from a moving vehicle?

\section{REFERENCES}

Alexander, J., Barham, P., and Black, I. (2002). Factors influencing the probability of an incident at a junction: results from an interactive driving simulator. Accident Analysis and Prevention 34, 779-792.

Brilon W., Koenig R., and Troutbeck, R. J. (1999). Useful estimation procedures for critical gaps Transportation Research part A, 33, 161-186.

Cody, D. (2005). Analysis of Field Test Data from an Instrumented Vehicle: Description of Left Turn Maneuvers for Intersection Safety Countermeasure Design. Proceedings of the TRB $84^{\text {th }}$ annual meeting, Washington, DC.

Chovan, J.D., Tijerina, L., Everson, J.H., Pierowicz, J.A., Hendricks, D.L. (1994). Examination of intersection, left turn across path crashes and potential IVHS countermeasures. Department of Transportation HS 808 164, 52 .

Hancock, P.A., Caird, J.K., Shekhar, S., and Vercruyssen, M. (1991). Factors influencing driver's left turn decisions. Proceedings of the Human Factors Society 35th Annual Meeting, Santa Monica, CA, 1139-1143

Nowakowski, C. (2006). A Preliminary Evaluation of a Driver- Infrastructure Interface for a Left-Turn Warning. Proceedings of the 50th Annual Meeting of the Human Factors and Ergonomics Society, Santa Monica, CA.

Ragland, D.R. and Zabyshny, A.A. (2003). Intersection Decision Support Project: Taxonomy of Crossing-Path Crashes at Intersections Using GES 2000 Data. U.C. Berkeley Traffic Safety Center. UCB-TSC-RR-2003-08.

Staplin, L. (1995). Simulator and field measures of driver age differences in left-turn gap judgments Transportation Research Record 1485, 49-55.

Ueno, H. and Ochiai, K. (1993). Analysis of accidents in right turns using a fuzzy logic simulation model (DOT-HS-807 990). Washington, DC: US Department of Transportation. 